\title{
BUILDING FACADE OBJECT DETECTION FROM TERRESTRIAL THERMAL INFRARED IMAGE SEQUENCES COMBINING DIFFERENT VIEWS
}

\author{
L. Hoegner * U. Stilla
}

Photogrammetry and Remote Sensing, Technische Universitaet Muenchen - (ludwig.hoegner, stilla)@tum.de

KEY WORDS: Urban, Combination, Terrestrial, Infrared, Sequences, Extraction, Texture, Building

\begin{abstract}
:
This paper discusses the automatic texturing of building facades from thermal infrared image sequences. A fully automatic method is presented to refine GPS based positions estimating relative orientations of the image sequences including a given building model in a bundle adjustment process. The resulting refined orientation parameters are used to extract partial facade textures from all images and all sequences. The resulting partial textures of every sequence are combined to get complete facade textures in the thermal infrared domain. Textures from different image sequences are combined for object detection and extraction. These sequences are acquired either at different times for different radiometric thermal behavior of facade objects or with different viewing directions for objects located before or behind the facade plane.
\end{abstract}

\section{INTRODUCTION}

\subsection{Motivation}

Today, IR inspections of buildings are still done in the image domain by analysing single images with image processing techniques (Balaras and Argiriou, 2002). The images are mainly taken manually for a single building only. However, mobile mapping systems are more and more used to carry out large area inspections for urban quarters or entire cities. The IR cameras can be mounted on a mobile terrestrial or a flying platform. Terrestrial images (Hoegner et al., 2007) taken from a vehicle can be used for documentation of frontal faces visible from the street level, while airborne images taken from an unmanned aerial vehicle (UAV) or helicopter can capture roofs. Using oblique view images inner yards can also be covered (Iwaszczuk et al., 2012). In contrast to images in the visible spectrum, textures in the thermal infrared spectrum can be used to extract objects under the surface like heating pipes and thermal leakages. First attempts for window extraction using grammars have already shown the potential of thermal infrared textures (Michaelsen et al., 2012). Combining IR images with 3D building information requires spatially referencing the data. Image data from different points in time, different platforms and multiple images (video sequences) can be fused. The assignment of the images to the 3D building model should be done automatically and can be achieved via texture mapping. As thermal infared cameras are from their construction limited to image dimensions between $320 \times 240$ and $1024 \times 768$ pixel, a small field of view is chosen to increase the geometric resolution of the textures on the facades. Due to this, single images show only small parts of facades. The complete facade is recorded within the sequence.

\subsection{State of the Art}

Today, automatic $3 \mathrm{~d}$ reconstruction and texture extraction is focused on high resolution images and image sequences from the visual spectrum. These methods are mainly using homologous points to link the images in a relative orientation and extract $3 \mathrm{~d}$ coordinates for the homologous points (Hartley and Zisserman, 2004). Some of these methods also include a self-calibration for

\footnotetext{
${ }^{*}$ Corresponding author
}

the camera (Agarwal et al., 2009; Longuet-Higgins, 1981; Maybank, 1993; Mayer et al., 2012). The resulting camera orientations and $3 \mathrm{~d}$ points have to be transferred from the model coordinate system to the global coordinate system either using external GPS/INS orientation information and / or a matching with given $3 \mathrm{~d}$ building models.

If a $3 \mathrm{~d}$ building model is already given in the beginning, a matching of the images and the building model can be done (Haralick et al., 1989, 1994; Horaud et al., 1989; Quan and Lan, 1999; Triggs, 1999; Zhi and Tang, 2002). This matching can be based on line segments, control points, or surfaces. The limitation of these methods is the accuracy and level od detail of the building model with respect to the scene visible in single images. If in an image there are only few parts of line segments, a correct matching of the image and the $3 \mathrm{~d}$ model is often not possible. If small facade objects should be extracted, a small field of view should be chosen as mention in subsection 1.1. As most existing building models do not have detailed geometry beyong facade planes, a direct matching of single images and $3 \mathrm{~d}$ building models is not possible.

Especially for thermal infrared images with their low image size, the number of feature points that can be used as homologous points is low compared to images in the visible spectrum. To find a good set of homologous points and remove outliers, the RANSAC algorithm (Fischler and Bolles, 1981) is used in most solutions, where random minimal sets of homologous points are used to estimate a relative orientation, that is checked against the other homologous points. A reduction to a minimum set of five corresponding points is possible for known interior orientation (Nister, 2004). This reduces the possibility of outliers in the minimum set of the RANSAC and increases the quality of the best relative orientation. An additional quality improvement is the extension of image pairs to image triplets (Hartley, 1997; Fitzgibbon and Zisserman, 1998; McGlone, 2004; Stewenius et al., 2005). Espacially for image sequences with a constant viewing direction, the trifocal tensor derived from image triplets increased significantly the stability of the relative orientation compared to image pairs using the fundamental matrix.

Nister's algorithm is adapted to extract $3 \mathrm{~d}$ points and textures of facades from image sets with various positions and viewing directions (Mayer, 2007). For wide baseline configuration the re- 
sulting $3 \mathrm{~d}$ points allow the extraction of windows (Reznik and Mayer, 2008). Introducing a semi-global matching (Hirschmller, 2008) dense point clouds can be generated from the relative oriented image sets (Mayer et al., 2012). This method is also applied to image sets from UAVs (Bartelsen and Mayer, 2010).

Combining the Five-point-algorithm (Nister, 2004) with plane sweeping (Yang and Pollefeys, 2003) a rough model of the scenery is calculated in close real time (Pollefeys et al., 2008). Necessary camera orientations are calculated using the relative movement of homologous points through the image sequence(Nister et al., 2006) and a Kalman filter using additional GPS/INS data (Grewal and Andrews, 2008).

Recording image sequences of building facades most of the homologous points are on the facade plane. This has a significant influence on the quality of the relative orientation as the reconstruction of the interior orientation is not possible from a planar scene (Maybank, 1993). For calibrated cameras with known interior orientation a planar scene has two solutions in general (Longuet-Higgins, 1986; Maybank, 1993). The geometrical correct solution can be found by searching the solution with all $3 \mathrm{~d}$ points in front of both cameras. For planar scenes the homography is an alternative way of orienting image pairs (Hartley and Zisserman, 2004; Pollefeys et al., 2002).

There is quite a limited number of works on transferring methods for geometric calibration and $3 \mathrm{~d}$ reconstruction to the thermal infrared domain. A geometric calibration including principal point, focal length, and radial distortion parameters has been investigated by some groups (Simmler, 2009; Luhmann et al., 2010; Lagela et al., 2011). 3d reconstruction and texture extraction in thermal infrared are applied for sets of images (Gehrke et al., 2013) and ordered image sequences (Hoegner et al., 2007). Both $3 \mathrm{~d}$ reconstruction and texturing are influenced by various conditions as the thermal radiation of facades depends on temperature differences between inside and outside, weather conditions, and materials. To overcome limitations in the $3 \mathrm{~d}$ accuracy of thermal infrared based $3 \mathrm{~d}$ points, a combination of thermal infrared cameras and $3 \mathrm{~d}$ recording systems like laserscanners (Borrmann et al., 2013) and time-of-flight cameras (Hoegner et al., 2014) is possible.

\section{METHODOLOGY}

The proposed concept is based on the assumption that a $3 \mathrm{~d}$ model of the recorded building is given containing $3 \mathrm{~d}$ vertex coordinates and triangulated polygon surfaces with given texture coordinates. The $3 \mathrm{~d}$ coordinates of the building are given in UTM. Instead of generating an isolated only relative oriented model, in this approach the image sequence is matched with the pre-known building model and measured camera path. Several matching steps are performed:

At first, a camera orientation is interpolated for every image of the sequence from the recorded GPS/INS data. Starting with the first image pair, homologies points are tracked through the images. Given the camera orientation and known camera calibration, the observed image coordinates are used to calculate $3 \mathrm{~d}$ coordinates in a bundle adjustment. Estimated $3 \mathrm{~d}$ points of a facade should be on or close to a polygon face of the given $3 \mathrm{~d}$ model. This constraint is added in the adjustment to minimize the distance of the extracted $3 \mathrm{~d}$ points to the polygon surface and remove outliers.
After this step, the corrected camera orientation is used to project the polygonal model into the images of the sequence to interpolate intensity values for the facade textures. To be able to combine several images of a facade for the final facade texture, the resolution of the outgoing texture is defined first. Caused by the strict order of the sequence partial occlusion of facades have to be taken into account. This procedure is repeated for every visible facade in every input image of the sequence. Additionally, for every pixel of the texture, the geometric resolution is stored.

The resulting complete texture is calculated by merging the pixel values of the partial textures. This is achieved by copying one over another in the order of the time stamps. Using an oblique view for the recorded sequence, every visible pixel of a texture has a higher geometric resolution than the texture before. To overcome remaining positioning errors, a line matching between the partial textures is included in this step.

The combination of textures from different times and viewing directions is then done based on the texture coordinates of the facades.

\subsection{Orientation of image sequences}

Given the known interior orientation of the thermal infrared camera from an initial geometric calibration (Simmler, 2009; Luhmann et al., 2010), SIFT features (Lowe, 2004) are tracked through the images of a sequence using cross-correlation. Figure 1 shows an example of features tracked in the sequence where the arrows point in the direction of the movement of the features between two images.

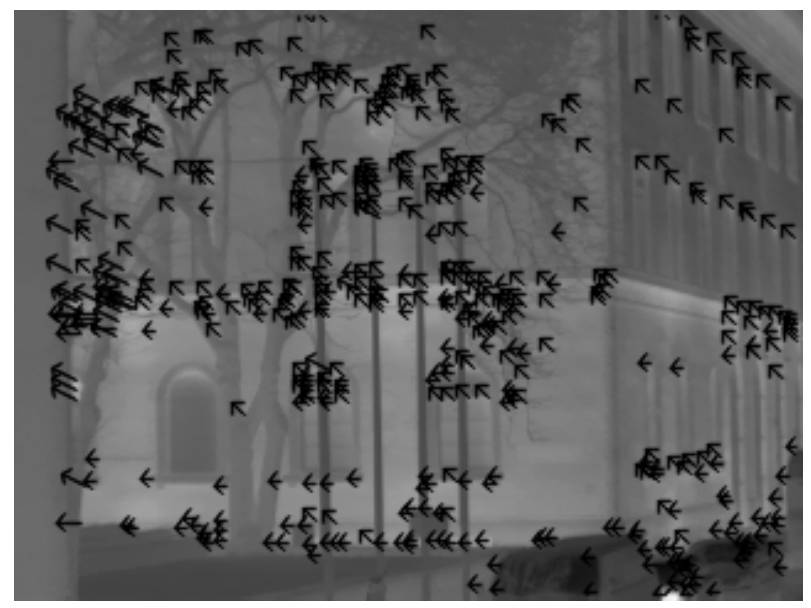

Figure 1. IR image with selected SIFT features, that have correspondences in the following image. Arrows show the moving direction of the points

Using the collinearity equations as basis in the bundle adjustment the observations are given as the image coordinates $\left(x_{i k}, y_{i k}\right)$ where $i$ is the numbering of homologous image points of an object point $\left(X_{i}, Y_{i}, Z_{i}\right)$ and $k \in[1, n]$ the numbering of the images $\left(B_{1} \ldots B_{n}\right)$ of the sequence. The object point coordinates are unknowns where an initial estimation is made from two images with the longest baseline. The interior orientation $\left(x_{0}, y_{0}, c_{x}, c_{y}\right)$ including radial distortion coefficients $\left(A_{1}, A_{2}\right)$ and the exterior orientations $\left(X_{0 k}, Y_{0 k}, Z_{0 k}, \omega_{k}, \phi_{k}, \kappa_{k}\right)$ of the images $\left(B_{1} \ldots B_{n}\right)$ are also unknowns with the calibration values and GPS/INS path as initial estimations.

The given $3 \mathrm{~d}$ building model is added as additional virtual observations. A $3 \mathrm{~d}$ object point $\left(X_{i}, Y_{i}, Z_{i}\right)$ of a facade has to be on 
the facade. If the facade plane is defined in Hesse normal form.

$$
\vec{r} \cdot \vec{n}-d=0
$$

then $\vec{n}$ is the normalized normal vector of the plane and $d$ the distance of the plane to the origin of the coordinate system. A $3 \mathrm{~d}$ object point $\mathrm{P}$ is on this plane, if its vector $\vec{r}=\left(X_{i}, Y_{i}, Z_{i}\right)^{T}$ fulfills equation 1 . This equation is now used as virtual observation for every facade plane.

The assignment of a $3 \mathrm{~d}$ object point to a facade plane is done calculating the intersection of the projection rays of the homologous image points $\left(x_{i k}, y_{i k}\right)$ through the perspective centers $\left(X_{0 k}, Y_{0 k}, Z_{0 k}, \omega_{k}, \phi_{k}, \kappa_{k}\right)$ and the facade planes. This intersection is calculated using

$$
g: \vec{r}=\overrightarrow{r_{0}}+\lambda \cdot \vec{u}
$$

where $\overrightarrow{r_{0}}$ is the position vector and $\vec{u}$ the direction. The position vector is the perspective center $\overrightarrow{r_{0}}=\left(X_{0}, Y_{0}, Z_{0}\right)^{T}$. The direction is the normalized vector between $(x, y)$ and the perspective center. All points $\vec{r}$ with $\lambda$ fulfilling the equation are on the projection ray. Combining equation 1 and 2 leads to

$$
\lambda=\frac{d-\overrightarrow{r_{0}} \cdot \vec{n}}{\vec{u} \cdot \vec{n}}
$$

The resulting $\lambda$ inserted in the projection equation results in the intersection points $\left(X_{i k}, Y_{i k}, Z_{i k}\right)$ of the projection ray and the facade plane. A $3 \mathrm{~d}$ point on a facade is an inlier, if it is a homologous point in at least to images where the intersections of the projection rays with the plane are close together. Homologous points where the intersection points are not within a defined variance are marked as outliers and removed from the observations. All observations are image coordinates of homologous points and thus taken as uncorrelated and with the same standard deviation for the stochastic model.

\subsection{Texture Extraction}

Given the refined image orientations of subsection 2.1, the polygons of the $3 \mathrm{~d}$ building model are projected into the images. For the facade textures a unified geometric resolution is defined. Given the texture coordinates of the four corners of a facade $P_{l o}=(0,0), P_{l u}=(m-1,0), P_{r u}=(m-1, n-1), P_{r o}=$ $(0, n-1)$ and there corresponding object coordinates $\left(X_{p}, Y_{p}, Z_{p}\right)$ the four corner points of the texture are projected into the recorded image using the collinearity equations. This results in four point correspondences between the texture image in texture coordinates and the recorded image in image coordinates. These four correspondences are used to calculate the four parameters of a rectification. The resulting parameters are then used to calculated image coordinates in the recorded image for every pixel of the texture and interpolated its intensity value using bilinear interpolation. Texture pixels without four neighboring pixels in the recorded image are left blank. Figure 2a shows an input image of the $3 \mathrm{~d}$ building model projected into the image space using the resulting parameters of the interior and exterior camera orientation from the orientation step. Due to oblique view, the geometric resolution decreases from the left to the right when the percentage of the facade in height is increasing (Fig. 2b). White areas have the highest geometric resolution, black areas the lowest. Non visible parts of the facade are marked in red. combination of several textures of the same facade extracted from several images shows the whole facade with a geometric resolution decreasing towards the roof (Fig. 2c+d).

From figure $2 \mathrm{c}$ it is obvious that if textures are generated in the order of the recording sequence, an extracted partial texture has a

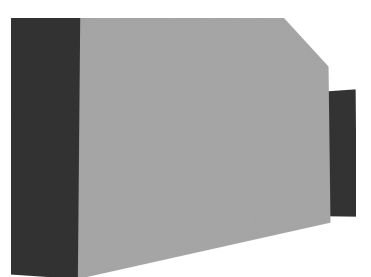

(a)

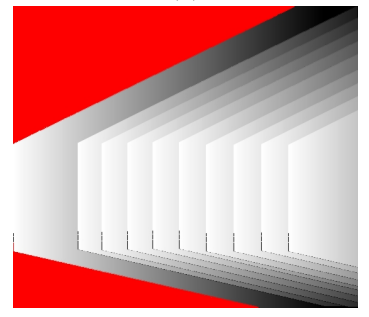

(c)

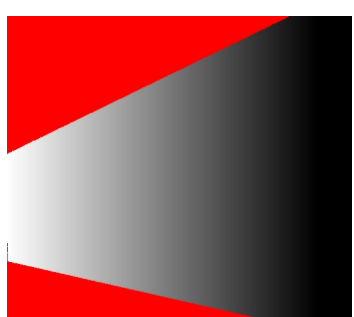

(b)

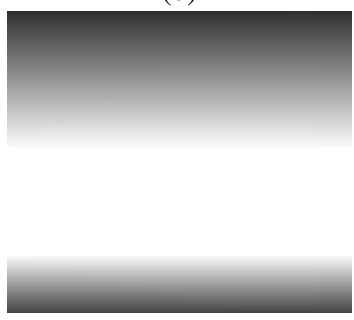

(d)
Figure 2. Geometric resolution of textures a) Oblique forward view of a facade b) Schematic view of the geometric resolution of a partial texture taken from an image in oblique forward looking view. white: high resolution, black: low resolution, red: not visible in the image c) resolution of a facade texture for a set of partial textures d) resolution for a facade texture from partial textures of an image sequence

higher geometric resolution for all visible parts than the textures before in the same sequence. Thus, textures are simple copied one over another in recording order for all pixels with an interpolated intensity value. For remaining projection errors a correction is applied by matching the partial texture to add to the existing texture using corresponding feature points.

This texture extraction scheme is valid for all visible facades even those perpendicular to the viewing direction. For backward viewing recordings, the order of the texture combination has to be flipped because now the geometric resolution decreases within the sequence as the camera is moving away from visible points.

\subsection{Combined analysis of textures from different views}

As mentioned in subsection 2.2, all facade textures are calculated with the same geometric resolution. So all textures of one facade from different image sequences have the same dimensions. Different texture are extracted for forward and backward view and for different times comparing evening and morning textures. The different textures are combined using an intensity vector with the intensities of the different textures for every texture pixel coordinate.

The combination of textures from different recording times but almost same viewing direction is used to analyze dynamic processes as different parts of facades show - depending on their materials - different cooling and warming behavior. As the radiation of surfaces may depend on the viewing direction, the almost same viewing direction is chosen for this texture combination.

The combination of textures from different viewing directions is used for disparity measurements (Fig. 3). In the texture extraction facades have been takes as flat. This leads to projection errors for object points before or behind the facade plane. For object points in the facade plane (Fig. 3a) the pixels in the to textures fall together. For object points before or behind the facade plane the projection error results in a disparity of the corresponding pixels in the textures in moving direction of the camera (Fig. 3b). 


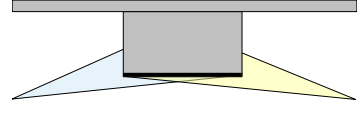

(a)

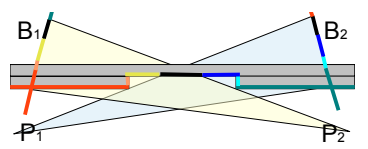

(b)
Figure 3. Occlusions caused by oblique view: a) Forward and backward recording geometry of a flat facade. b) Forward and backward recording geometry of a window. Caused by the oblique views both images show different parts of the window and the inner part is shifted in both images B1 and B2

The general setup of the texture generation allows the combination of textures extracted from different sensors in the visible, near infrared, midwave thermal infrared and longwave thermal infrared. The combined analysis of textures from these spectral bands is still ongoing.

The combined analysis of textures from different views is used for leakage detection. In thermal infrared images, leakages appear as areas with higher emission values than the surrounding surface. Other facade elements show a similar radiometric behavior. Ledges are exposed objects on a facade an thus loose more energy than the facade plane. Reveals store at their upper ledge warm rising air. Both effects look similar to leakages. And different material can cause different intensities for the same temperature. It is assumed that material changes on a facade either concentrate on small areas and belong to facade objects or divide the facade into a few big, extensive areas. If objects before and behind the facade plane are masked out by identifying pixels with disparities in the two textures, the remaining facade textures contains the homogenous facade plane. Small segments in this remaining textures with a significant intensity difference to the local neighborhood of the facade are then candidates for leakages.

A histogram of the remaining texture pixels is used to find the $\max (p(g))$ for all intensities $g$. Under the assumption, that the majority of the texture pixels shows facade plane without leakage, this maximum is the mean temperature of the facade plane without leakages and objects. It is further assumed that the intensities of possible leakages are further local maxima in the histogram. A region growing is used to group pixels with similar intensity values. Adjacent regions with similar intensities are merged. In the end, there remains a big region of the facade plane and leakages as small segments.

\section{EXPERIMENTS}

Our experiments have been taken using a SC3000 thermal infrared camera with a detector of 320x240 pixels and a temperature resolution of $0.02 \mathrm{~K}$. Image sequences have been taken after sunset and before sunrise. Figure 4 shows some images out of four sequences in forward view showing different facades of a building complex. One sequence shows a group of facades with different orientation and occlusions (Fig. 4a). The other scenes show several building parts in the same plane but with different structures (Fig. 4b-d). The sequence was recording with 50 frames per second. This allows a very good feature tracking due to only small movements between the images. For the $3 \mathrm{~d}$ reconstruction every 10th image was taken to reduce the computational effort and guarantee a $3 \mathrm{~d}$ base necessary for the initial $3 \mathrm{~d}$ point estimation.

\subsection{Texture extraction}

The estimated $3 \mathrm{~d}$ point coordinates and camera orientations after the bundle adjustment are mainly depending on the number

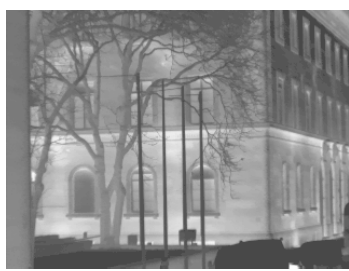

(a)

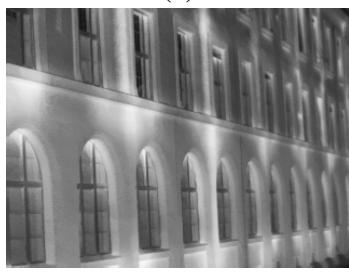

(c)

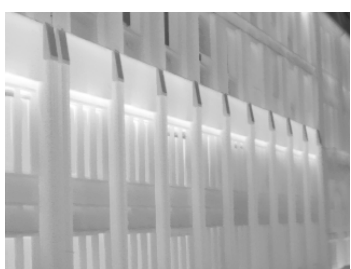

(b)

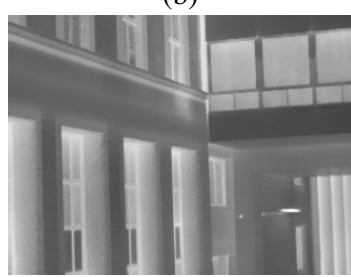

(d)
Figure 4. Overview over recorded scenarios with the SC3000 thermal infrared camera looking forward a) Several facades with different orientation and occlusions. b) Three building parts in the same facade plane but with different structure, the modern one with a repetitive pattern in the windows. c) Several small building parts in the same facade plane d) Several building parts of different type in the same facade plane. Bridges occluding parts of the facades.

and quality of the feature points that are tracked through the sequences. In table 1 the detected SIFT features in thermal image sequences are compared to a video sequence recorded at the same time in original resolution $(768 \times 576)$ and down sampled to the same resolution as the thermal images $(320 \times 280)$.

\begin{tabular}{|c|c|c|c|}
\hline & \multicolumn{3}{|l|}{ SIFT } \\
\hline & Video 768 & Video 320 & IR 320 \\
\hline \# Interest points & 1246.2 & 531.4 & 168.4 \\
\hline \multicolumn{4}{|l|}{1 frame per second } \\
\hline \# homologous points & 287.2 & 124.7 & 31.9 \\
\hline tracking time in seconds & 11.6 & 11.6 & 10.4 \\
\hline sum of squared differences & 0.006 & 0.011 & 0.025 \\
\hline \multicolumn{4}{|l|}{5 frames per second } \\
\hline \# homologous points & 683.3 & 244.6 & 129.2 \\
\hline tracking time in seconds & 11.8 & 11.8 & 10.5 \\
\hline sum of squared differences & 0.005 & 0.009 & 0.012 \\
\hline
\end{tabular}

Table 1. Quality of Feature points. Mean values over all sequences. Number of features in images in the visual spectrum (original resolution and down sampled) compared to number of features in thermal infrared.

Due to the lower contrasts and lack of small details the number of detected features is much lower in the thermal images than in the visible spectrum. Nevertheless a sufficient number of features is tracked through the sequence. The differences in the tracking time result from the oblique view and the lower contrast in the thermal scene as features are detected first not directly when they first appear in the image but only after several images of the sequence when their size allows a detection. The sum of squared differences (SSD) indicates how similar two homologous points are in different images. A SSD of 0 means they are totally equal.

An exemplary $3 \mathrm{~d}$ point cloud and camera path can be seen in figure 5 . The $3 \mathrm{~d}$ points are grouped along the window rows where the thermal images show the highest gradients. In the center the tree is extracted. Its points are not used for the orientation improvement as they are to far away from a $3 \mathrm{~d}$ model surface but are calculated in the bundle adjustment.

Performing a bundle adjustment on the image sequences $4 \mathrm{a}-4 \mathrm{~d}$ 


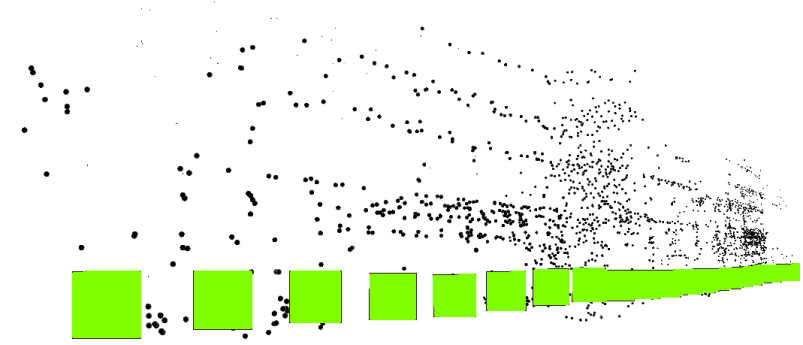

Figure 5. 3d point cloud and estimated camera path.

without pre-knowledge at 5 frames per second, only the feature points and interior orientation are used as observations where the object coordinates and exterior orientations of the cameras are the unknowns. The resulting standard deviations for the unkowns are listed in table 2 .

Comparing these standard deviation to the standard deviations for the unknowns including pre-knowledge of the building model (table 3) show small improvements on the accuracy of the bundle adjustment.

\begin{tabular}{|l|l|l|l|l|}
\hline relative orientation & $4 \mathrm{a}$ & $4 \mathrm{~b}$ & $4 \mathrm{c}$ & $4 \mathrm{~d}$ \\
\hline$\sigma_{x y}[\mu \mathrm{m}]$ & 0.3857 & 12.74 & 0.9486 & 14.71 \\
$\sigma_{X Y Z}[\mathrm{~m}]$ & 0.06855 & 0.3627 & 0.1068 & 0.3815 \\
$\sigma_{X 0}[\mathrm{~m}]$ & 0.1285 & 0.2512 & 0.08374 & 0.3486 \\
$\sigma_{Y 0}[\mathrm{~m}]$ & 0.04055 & 0.1856 & 0.2731 & 0.4502 \\
$\sigma_{Z 0}[\mathrm{~m}]$ & 0.02063 & 0.05744 & 0.06718 & 0.0815 \\
$\sigma_{\omega 0}[$ gon $]$ & 0.03016 & 0.05230 & 0.6114 & 1.029 \\
$\sigma_{\phi 0}[$ gon] & 0.06945 & 0.2041 & 0.09485 & 0.5176 \\
$\sigma_{\kappa 0}$ [gon] & 0.2259 & 0.4583 & 0.5834 & 1.923 \\
\hline
\end{tabular}

Table 2. Accuracy of the relative orientation without preknowledge

\begin{tabular}{|l|l|l|l|l|}
\hline absolute orientation & $4 \mathrm{a}$ & $4 \mathrm{~b}$ & $4 \mathrm{c}$ & $4 \mathrm{~d}$ \\
\hline$\sigma_{x y}[\mu \mathrm{m}]$ & 0.3534 & 1.529 & 0.9185 & 1.405 \\
$\sigma_{X Y Z}[\mathrm{~m}]$ & 0.05944 & 0.1910 & 0.09583 & 0.1748 \\
$\sigma_{X 0}[\mathrm{~m}]$ & 0.04454 & 0.06776 & 0.04969 & 0.03390 \\
$\sigma_{Y 0}[\mathrm{~m}]$ & 0.02294 & 0.01750 & 0.1005 & 0.1972 \\
$\sigma_{Z 0}[\mathrm{~m}]$ & 0.009547 & 0.03065 & 0.06421 & 0.04909 \\
$\sigma_{\omega 0}$ [gon] & 0.02323 & 0.3148 & 0.5981 & 0.6936 \\
$\sigma_{\phi 0}$ [gon] & 0.02196 & 0.08770 & 0.05319 & 0.4930 \\
$\sigma_{\kappa 0}$ [gon] & 0.2016 & 0.3806 & 0.5991 & 1.522 \\
\hline
\end{tabular}

Table 3. Accuracy of absolute orientation including preknowledge of the building model

Whereas the improvements for $4 \mathrm{a}$ and $4 \mathrm{c}$ are quite small, the imporvements for $4 \mathrm{~b}$ and $4 \mathrm{~d}$ are significant. $4 \mathrm{~b}$ and $4 \mathrm{~d}$ have very high standard deviations in the relative orientation compared to $4 \mathrm{a}$ and $4 \mathrm{c}$. This is caused by the homogenous structure of the facades (4b) and the occlusions (4d). Both scenarios show less homogeneous points that can be tracked through the sequence and more false correspondences.

Including the building model allows to remove more false correspondences which helps in sequence $4 \mathrm{~d}$. In sequence $4 \mathrm{~b}$ the known facade plane assists in estimating the movement of the features through the sequences.

Despite the standard deviation, the accuracy of the oriented sequence to the building model is necessary for an accurate texture extraction. Whereas in the bundle adjustment including the building model the sequence is already matched to the building model, in the relative orientation without pre-knowledge, the sequence and the extracted $3 \mathrm{~d}$ object points have to be matched to the building model, a step, that introduces additional deviations of 0.05 meters in $\mathrm{X}, \mathrm{Y}$, and $\mathrm{Z}$ and 0.02 gon in $\omega, \phi, \kappa$.

Figure 6 shows one example texture extracted after orientation with pre-knowledge. As the partial textures (fig. $6 a+b)$ are extracted from an oblique view, the spatial resolution is not constant. It is getting lower from the left to the right. Due to this the textures are blurred at the right. On the other hand, on the left side only the first and parts of the second floor are visible. The third floor is visible only in the low resolution right part. A correlation is done for every added texture to remove remaining positioning errors (fig. 6c). Combining several of these partial textures leads to the complete texture given in figure $6 \mathrm{~d}$. The total facade is visible in the texture but with different geometric resolutions. For the first and second floor the resolution is higher than for the third floor. The big bus standing in front of the building could only be removed to a small part because it was occluding the facade in all images.

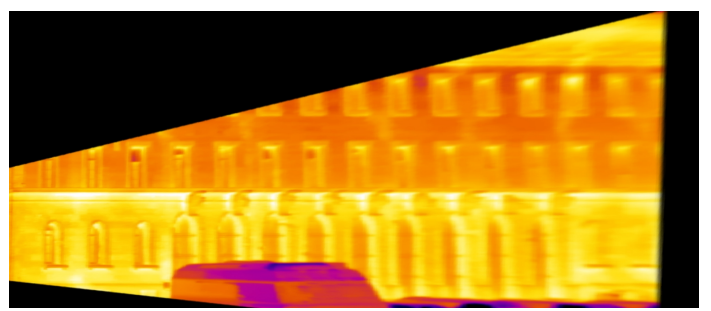

(a)

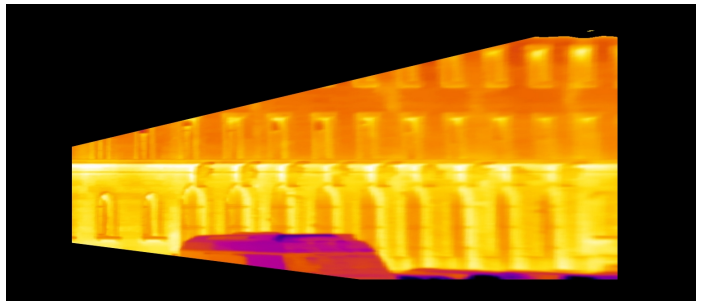

(b)

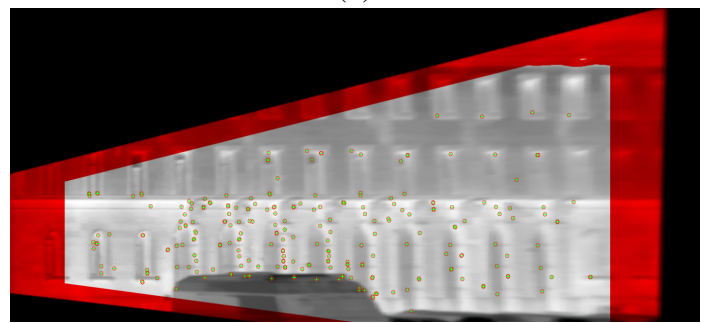

(c)

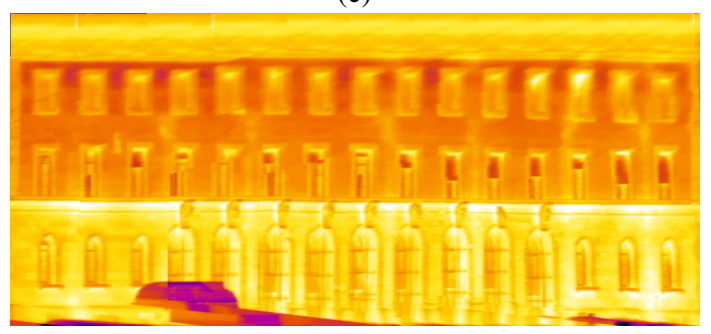

(d)

Figure 6. Facade texture combined from several partial textures. a)+b) Two partial textures extracted from the same image sequence. c) Co-registered overlay of (a) and (b). Green: Interest points in image (a). Red: Interest points in image (b). d) Final composed texture. 


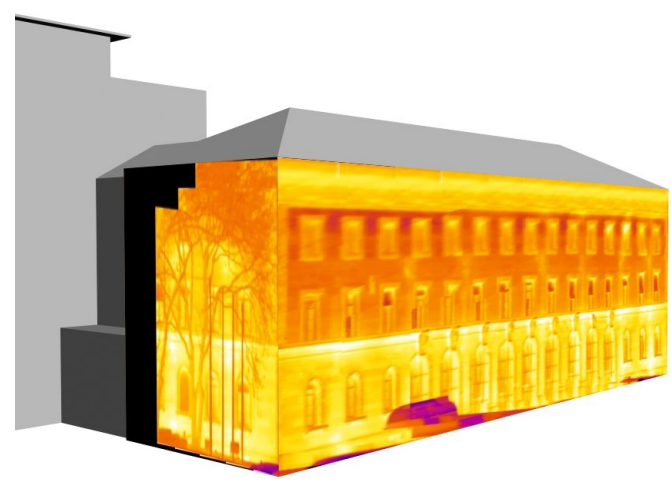

Figure 7. $3 \mathrm{~d}$ building model with facade textures

\subsection{Texture analysis}

A combination of textures from forward and backward viewing images is possible, if the time difference between both recordings is small. In the experiments this is done by driving along the facades first in forward view followed by the backward view (fig. $8 \mathrm{a}+\mathrm{b})$. The resulting textures are co-registered to compensate remaining errors in the texture projection process. In an algebraic transformation the difference of both textures is calculated pixel per pixel (fig. 8c). The left window in the middle row (fig. 8c, A) shows a higher temperature in the forward view. This window was open during the forward recording and closed before the backward recording. A car is standing in front of the building. Due to the oblique view, this car occludes a part of the facade in the forward view but not in the backward view (fig. 8c, B). For the windows and stone elements (fig. 8c, C) it can be seen that they show both a red and a blue border. This happens because windows and stone elements are not in the facade plane and thus are projected at a wrong position in the texture which leads to a disparity between the texture from the forward view and the texture from the backward view. Additionally both types show another intensity than the facade plane.

In contrast to the combination of textures from forward and backward viewing images a combination of textures from image sequences recorded in the evening and in the morning (Fig. 9a+b) allows the observation of dynamic processes over time. Difference texture of the coregistered textures from the evening and the morning shows the cooling of the facade over night (Fig. 9c). The color bar indicates the temperature difference in degree Celsius. The transfer from intensity values to temperatures is done using the radiometric calibration of the cameras and an emissivity value of $95 \%$. The biggest cooling effects can be seen again at the window that was open during the first sequence record and closed during the second record and at the car in front.

Leakages on facades are defined as regions with a higher intensity than the facade plane in the thermal infrared. This description is also true for other facade elements like windows. To detect the leakages, in a first step, other elements have to be masked out. As shown in figure 8 elements that are not in the facade plane cause a disparity in the difference texture of the texture from forward and backward view. A segmentation is used to generate a binary mask to detect these parts of the texture (Fig. 10a). Using a region growing and morphological operators on the segments masked in the previous steps, the resulting segments are used to masked out these parts of the image (Fig. 10b).

Figure 10c shows the result of the leakage detection. In the remaining part of the texture that has not been masked out a histogram based threshold is used to distinguish the facade (global maximum in the histogram) and the leakages. Windows and other

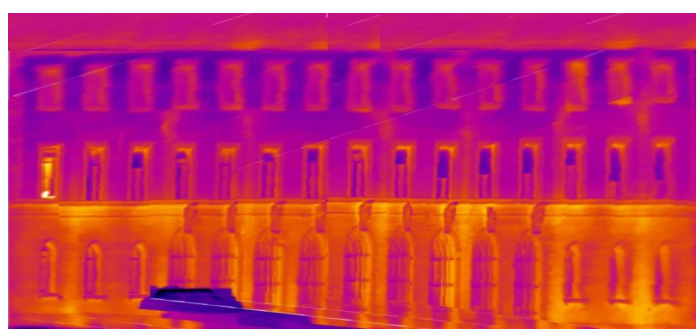

(a)

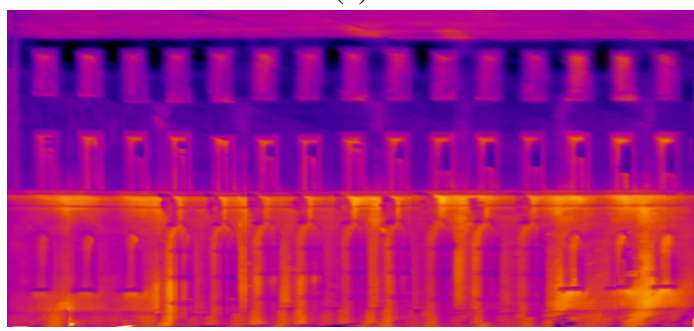

(b)

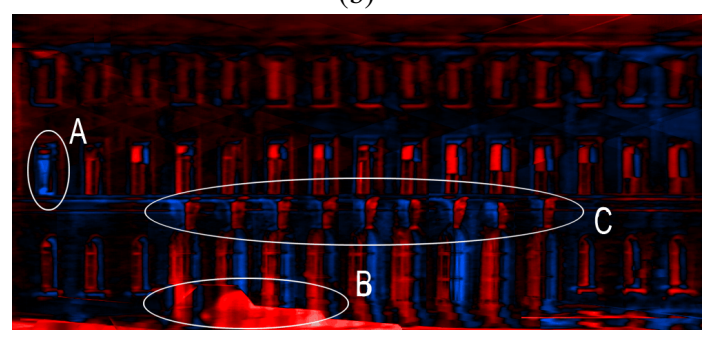

(c)

Figure 8. Combination of texture from forward and backward viewing images. a) texture from forward viewing images. b) texture from backward viewing images. c) Difference image after texture co-registration. Black: constant intensity in both images. Red: higher intensity in backward viewing texture. Blue: higher intensity in forward viewing texture. A) Windows that was close after the forward viewing image recording. B) Car in front of the facade that is projected incorrect into both textures. C) Stone elements standing in front of the facade plane.

$3 \mathrm{~d}$ structures are ignored for the leakage detection. Six out of seven heating pipes in the first floor have been found correctly. In the ground floor four heating pipes are detected. The heating pipes between the big windows in the middle are missing because they are to close to the windows and masked out. Additionally, a few small leakages close to windows have been detected.

\section{DISCUSSION AND OUTLOOK}

Different aspects influence the quality of the extracted textures. The accuracy of the $3 \mathrm{~d}$ reconstruction is limited compared to image sequences in the visual spectrum. One aspect is the lower image resolution that causes a higher discretization of the scene and reduces the number of details that can be matched within the sequence. On the other hand the radiometric behavior of object in the thermal infrared leads to blurred edges and a low number of intensity changes and details. Homologous points are rarer than in the visual spectrum and worse locatable. A second aspect is the geometric calibration of the cameras. Because of the two limitations already mentioned, the calibration accuracy is also limited compared to cameras in the visual spectrum.

The Quality of the initial GPS positions is limited in urban scenarios caused by mirroring and occlusion of the GPS signals. The initial values have quite an influence on the final results as initial assignments of homologous points to facades influence the 


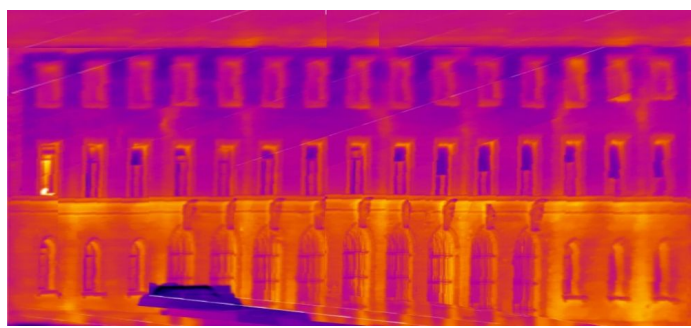

(a)

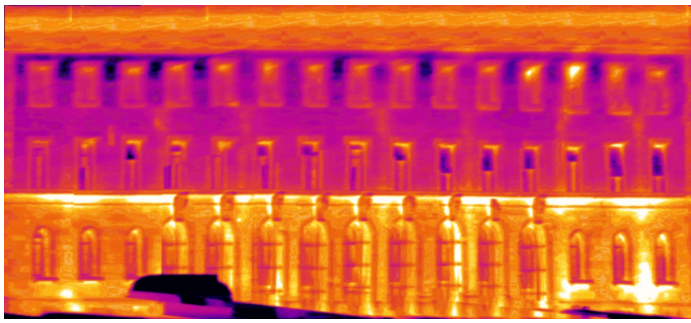

(b)

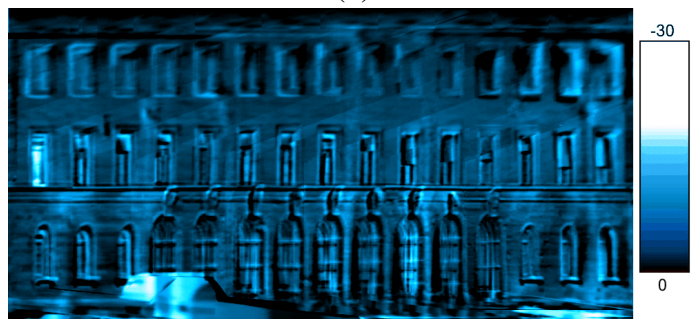

(c)

Figure 9. Cooling process in a difference texture from texture from a) evening and b) morning. c) Difference texture showing the cooling over night. Color scale in degree Celsius. Highest cooling at the open window and the car.

weightings in the iterative process. The quality of the building model itself is also a limiting factor. Building in level-of-detail 1 are only block models. In these models, the overhang at the roofs is not modeled. This causes two possible errors. If no overhang is modeled, the facades can either be set to the position of the footprint. Then, the position of the facades is correct, but the overhang is projected onto the facade. This causes misalignment of the objects on the facade like windows and heating pipes. If the building model is generated from aerial images, the roofs outline is taken as footprint and the position of the facades is incorrect in the model. In this case, the bundle adjustment tries to move the camera path which very often works but not necessarily. The number of visible elements in the thermal infrared influences the quality of the position refinement. On facades with only few objects or only repetitive patterns, the quality of the refinement is significantly reduced. If this falls together with bad initial GPS positions, the method can totally fail. The texture extraction is quite sensitive to errors in the viewing direction estimation. The combination of relative orientation and $3 \mathrm{~d}$ model knowledge reduces these errors significantly. Remaining errors between to partial textures.

Further steps in the processing of the textures are windows extraction, leakage detection and parameter estimation for facades. The given method can be used to record the building facades in whole streets to get first view on the quality of the thermal behavior of the buildings. Searching objects like windows, heating pipes, or leakages in building textures instead of images allows a $3 \mathrm{~d}$ geo-referencing and observation of thermal relevant objects i.e. in a building information model. The evaluation of time series and changes i.e. before and after a thermal optimization, summer vs. winter, evening vs. morning are possible in an automatic way.

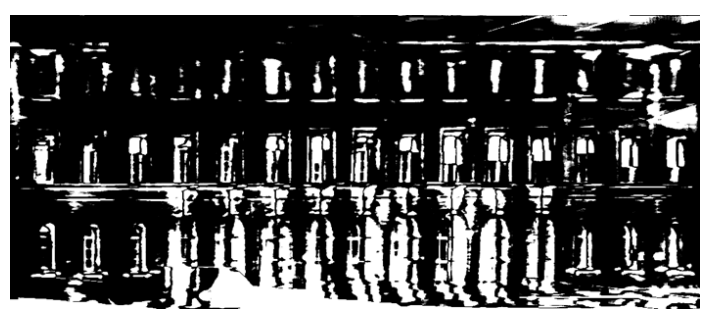

(a)

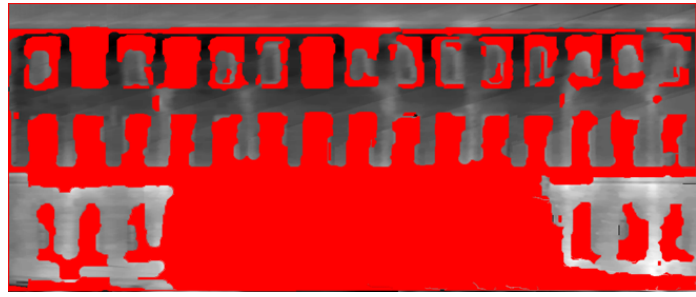

(b)

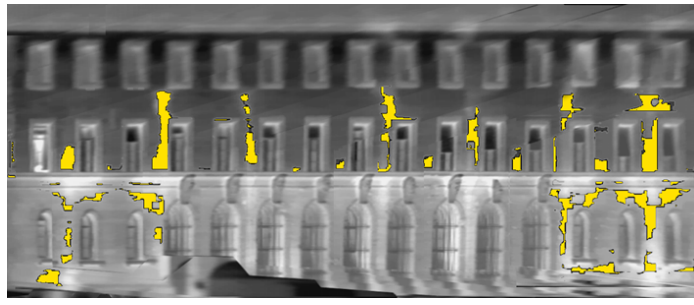

(c)

Figure 10. Leakage detection a) Disparity from difference texture of textures from forward and backward view. b) Mask after post processing with region growing and morphological operators. All windows are masked out. c) Marked leakages (yellow) overlayed the original texture. Windows and other $3 \mathrm{~d}$ objects are not detected as leakages. Most of the heating pipes are detected as leakages.

\section{ACKNOWLEDGEMENTS}

The authors thank Dr. Clement, Dr. Schwarz and Mr. Kremer of Fraunhofer IOSB, Ettlingen, for their assistance during the recording campaign.

\section{References}

Agarwal, S., Snavely, N., Simon, I., Seitz, S. and Szeliski, R., 2009. Building rome in a day. In: Proceedings of the International Conference on Computer Vision.

Balaras, C. and Argiriou, A., 2002. Infrared thermography for building diagnostics. Energy and Buildings 34, pp. 171-183.

Bartelsen, J. and Mayer, H., 2010. Orientation of image sequences acquired from uavs and with gps cameras. Surveying and Land Information Science 70(3), pp. 151-159.

Borrmann, D., Houshiar, H., Elseberg, J. and Nchter, A., 2013. Vom kombinieren von 3d-modellen mit farb- und temperaturinformationen. Photogrammetrie - Laserscanning - Optische 3D-Messtechnik. Beitrge der Oldenburger 3D-Tage 2013 pp. $246-253$

Fischler, M. and Bolles, R., 1981. Random sample consensus: A paradigm for model fit-ting with applications to image analysis and automated cartography. Communications of the ACM 24(6), pp. 381-395.

Fitzgibbon, A. and Zisserman, A., 1998. Automatic camera recoveryfor closed or open image sequences. Lecture Notes in Computer Science. 
Gehrke, R., Trabold, D., Greiwe, A. and Abel, J., 2013. 3dmodellierung eines bauwerkes aus rgb- und thermalaufnahmen fr das facility managment. Photogrammetrie - Laserscanning - Optische 3D-Messtechnik. Beitrge der Oldenburger 3D-Tage 2013 pp. 254-263.

Grewal, M. and Andrews, A., 2008. Kalman Filtering Theory and Practice. John Wiley \& Sons. ISBN 0470173661.

Haralick, R., Joo, H., Lee, C., Zhuang, X., Vaidya, V. and Kim, M., 1989. Pose estimation from correspondence point data. SMC 19(6), pp. 1426-1446.

Haralick, R., Lee, C., Ottenberg, K. and Nlle, M., 1994. Review and analysis of solutions of the three point perspective pose estimation problem. International Journal of Computer Vision 13(3), pp. 331-356.

Hartley, R., 1997. Lines and points in three views and the trifocal tensor. International Journal of Computer Vision 22(2), pp. 125-140.

Hartley, R. and Zisserman, A., 2004. Multiple View Geometry in Computer Vision. Cambridge University Presss. ISBN 0521540518

Hirschmller, H., 2008. Stereo processing by semiglobal matching and mutual information. IEEE Transactions on Pattern Analysis and Machine Intelligence 30(2), pp. 328-341.

Hoegner, L., Hanel, A., Weinmann, M., Jutzi, B., Hinz, S. and Stilla, U., 2014. Towards people detection from fused timeof-flight and thermal infrared images. In: Photogrammetric Computer Vision 2014 - PCV2014, Vol. XL-3, pp. 121-126.

Hoegner, L., Kumke, H., Meng, L. and Stilla, U., 2007. Automatic extraction of textures from infrared image sequences and database integration for $3 \mathrm{~d}$ building models. PFG Photogrammetrie - Fernerkundung - Geoinformation 2007(6), pp. 459468.

Horaud, R., Conio, B., Leboulleux, O. and Lacolle, B., 1989. An analytic solution for the perspective 4-point problem. Computer Vision, Graphics and Image Processing 1989(47), pp. 33-44.

Iwaszczuk, D., Hoegner, L., Schmitt, M. and Stilla, U., 2012. Line based matching of uncertain $3 \mathrm{~d}$ building models with ir image sequences for precise texture extraction. PFG Photogrammetrie - Fernerkundung - Geoinformation 2012(5), pp. 511-521.

Lagela, S., Gonzlez-Jorge, H., Armesto, J. and Arias, P., 2011. Calibration and verification of thermographic cameras for geometric measurements. Infrared Physics \& Technology 54, pp. 92-99.

Longuet-Higgins, H., 1981. A computer algorithm for reconstruction of a scene from two projections. Nature 203, pp. 133135 .

Longuet-Higgins, H., 1986. The reconstruction of a plane surface from two perspective projections. In: Proceedings of the Royal Society of London. Series B, Biological Sciences, Vol. 227(1249), pp. 399-410.

Lowe, D., 2004. Distinctive image features from scale-invariant keypoints. International Journal of Computer Vision 60(2), pp. 91-110.

Luhmann, T., Ohm, J., Piechel, J. and Roelfs, T., 2010. Geometric calibration of therographic cameras. International Archives of Photogrammetry, Remote Sensing and Spacial Information Sciences 38(5), pp. 411-416.
Maybank, S., 1993. Theory of Reconstruction from Image Motion. Springer Verlag. ISBN 0387555374.

Mayer, H., 2007. 3d reconstruction and visualization of urban scenes from uncalibrated wide-baseline image sequences. PFG Photogrammetrie - Fernerkundung - Geoinformation 2007(3), pp. 167-176.

Mayer, H., Bartelsen, J., Hirschmueller, H. and Kuhn, A., 2012. Dense $3 \mathrm{~d}$ reconstruction from wide baseline image sets. In: F. Dellaert, J.-M. Frahm, M. Pollefeys, L. Leal-Taixe and B. Rosenhahn (eds), Outdoor and Large-Scale Real-World Scene Analysis. 15th International Workshop on Theoretical Foundations of Computer Vision, Dagstuhl Castle, Germany, June 26 - July 1, 2011, Vol. 7474, Springer, Berlin, pp. 285304.

McGlone, C., 2004. Manual of Photogrammetry. ASPRS. ISBN 1570830711

Michaelsen, E., Iwaszczuk, D., Sirmacek, B., Hoegner, L. and Stilla, U., 2012. Gestalt grouping on faade textures from ir image sequences: Comparing different production systemse. In: International Archives of Photogrammetry, Remote Sensing and Spatial Information Science, Vol. 39/B3, pp. 303-308.

Nister, D., 2004. An efficient solution to the five-point relative pose problem. IEEE Transactions on Pattern Analysis and Machine Intelligence 26(6), pp. 756-777.

Nister, D., Naroditsky, O. and Bergen, J., 2006. Visual odometry for ground vehicle applications. Journal of Field Robotics 23(1), pp. 3-20.

Pollefeys, M., Nister, D., Frahm, J., Akbarzadeh, A., Mordohai, P., Clipp, B., Engels, C., Gallup, D., Kim, S., Merrell, P., Salmi, C., Sinha, S., Talton, B., Wang, L., Yang, Q., Stewnius, H., Yang, R., Welch, G. and Towles, H., 2008. Detailed realtime urban $3 \mathrm{~d}$ reconstruction from video. International Journal of Computer Vision 78(2-3), pp. 143-167.

Pollefeys, M., Verbiest, F. and Gool, L. V., 2002. Surviving dominant planes in uncalibrated structure and motion recovery. In: Proceedings of the ECCV 2002. Part II, Vol. 2351, Springer, Heidelberg, pp. 837-851.

Quan, L. and Lan, L., 1999. Linear n-point camera pose determination. IEEE Transactions on Pattern Analysis and Machine Intelligence 21(8), pp. 774-780.

Reznik, S. and Mayer, H., 2008. Implicit shape models, selfdiagnosis, and model selection for $3 \mathrm{~d}$ facade interpretation. PFG Photogrammetrie - Fernerkundung - Geoinformation 2008(3), pp. 187-196.

Simmler, C., 2009. Entwicklung einer Messanordnung zur geometrischen Kalibrierung von Infrarot-Kameras. Bachelorarbeit der Technischen Universitt Mnchen.

Stewenius, H., Schaffalitzky, F. and Nistr, D., 2005. How hard is 3 -view triangulation really? In: Proceedings of the Tenth IEEE International Conference on Computer Vision, Vol. 2005(1), pp. 686-693.

Triggs, B., 1999. Camera pose and calibration from 4 or 5 known $3 \mathrm{~d}$ points. In: Proceedings of the Seventh IEEE International Conference on Computer Vision, Vol. 1999(1), pp. 278-284.

Yang, R. and Pollefeys, M., 2003. Multi-resolution real-time stereo on commodity graphics hardware. In: IEEE Computer Society Conference on Computer Vision and Pattern Recognition, Vol. 2003, pp. 211-217.

Zhi, L. and Tang, J., 2002. A complete linear 4-point algorithm for camera pose determinatio. MM Research Reprints 21 , pp. 239-249. 